\title{
Retroperitoneal High Grade Undifferentiated Pleomorphic Sarcoma as a Second Metachronous Neoplasm Following Intracranial Meningioma
}

\author{
Armin Kamyab, Michael John Jacobs* \\ Department of Surgery, Providence Hospital and Medical Centers, Southfield, USA. \\ Email: *mjjacobs@pol.net
}

Received July 26 ${ }^{\text {th }}$, 2011; revised August 23 ${ }^{\text {rd }}$, 2011; accepted September $15^{\text {th }}, 2011$.

\begin{abstract}
Introduction: Meningiomas and retroperitoneal sarcomas are two distinct rare tumors, with an incidence of 2 and 0.4 cases per 100,000 respectively. Case Report: We report the case of a 39 year old woman with a history of intracranial meningioma who was found two months later to have a large retroperitoneal sarcoma on CT scan after presenting to the emergency department complaining of abdominal pain. Pathologic evaluation was consistent with a high grade undifferentiated sarcoma. Discussion: It is highly unusual for a 39 year old otherwise healthy patient to present with two rare, seemingly unrelated tumors. It is our suspicion that there is a genetic component involved, however to date no association has been described in the literature between intracranial meningiomas and extracranial sarcomas. Conclusion: Besides being the first report of a retroperitoneal sarcoma occurring as a second metachronous neoplasm following an intracranial meningioma, we suspect that there is a genetic component involved. Further studies may indicate a genetic mutational link.
\end{abstract}

Keywords: Meningioma, Sarcoma, Genetic

\section{Introduction}

Meningiomas and retroperitoneal sarcomas are two distinct rare tumors, with an incidence of 2 and 0.4 cases per 100,000 respectively.

We present the case of a 39 year old female who was found after complaining of worsening headaches to have an intracranial meningioma. She underwent successful surgical resection, and two months later was found to have a large retroperitoneal sarcoma after presenting to the emergency department with complaints of left sided abdominal pain.

Symptomatic meningiomas warrant surgical resection and the prognosis parallels the histological grade. The mainstay of treatment for retroperitoneal sarcomas is complete surgical resection with microscopically negative margins, although the vast majority of patients experience recurrence despite successful resection.

There has been no published genetic association between intracranial meningiomas and extracranial sarcomas. Further studies may indicate a genetic mutational link.

\section{Case Report}

We report the case of a 39 year old woman who was otherwise healthy, who presented initially with complaints of increasing headaches. During her work up, an MRI of the brain was performed which demonstrated a homogeneously enhancing extra-axial mass consistent with a meningioma. There was significant mass effect, and the patient therefore underwent a right frontoparietal craniotomy with resection of the meningioma. Pathologic evaluation confirmed the diagnosis of meningioma, grade 1 . Patient tolerated the procedure well and was discharged from that admission in a stable condition. The patient had not received chemotherapy or radiation therapy.

Two months later, the patient presented to our emergency department with complaints of left sided abdominal pain and weight loss. On examination, a mass was palpable in the left abdomen. A CT scan of the abdomen and pelvis was performed which demonstrated a large centrally necrotic mass in the left with local mass effect, highly suspicious for malignancy (Figures 1 and 2). A CT guided biopsy was performed which demonstrated a 


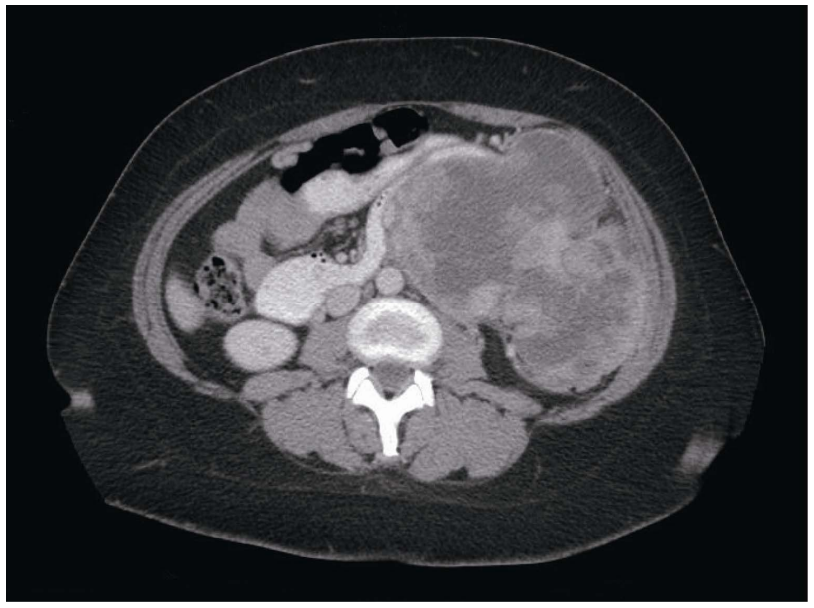

Figure 1. Axial CT scan demonstrating a large heterogeneous mass with central necrosis in the left abdomen, involving surrounding structures.

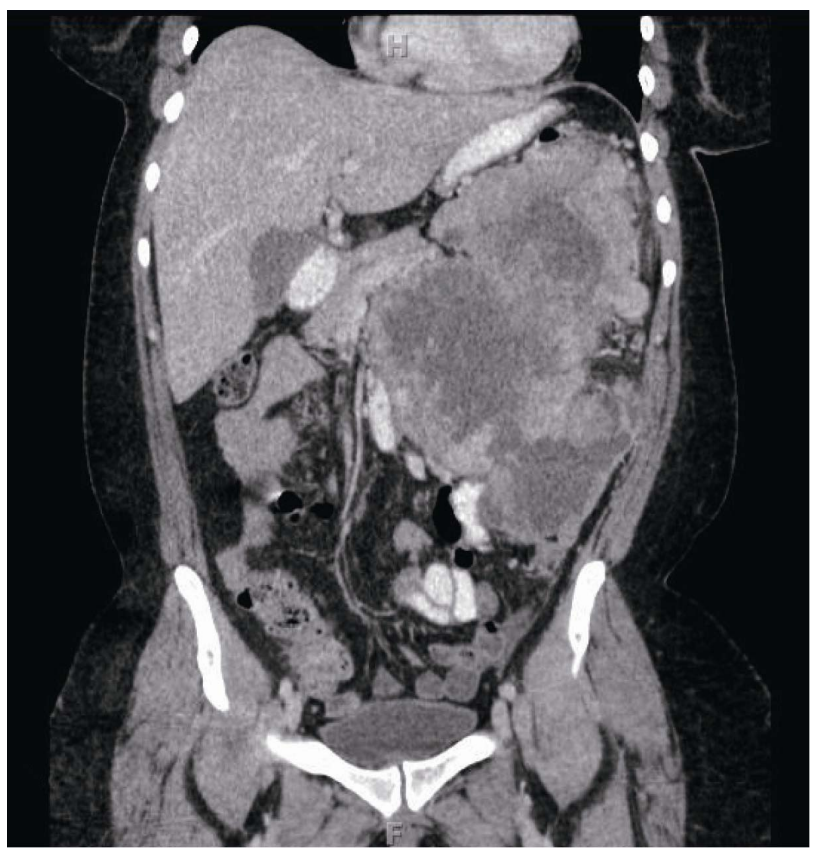

Figure 2. Coronal CT scan demonstrating a large heterogeneous mass with central necrosis in the left abdomen, involving surrounding structures.

spindle cell neoplasm. An exploratory laparotomy revealed a large necrotic ruptured malignant appearing neoplasm. This was resected en bloc with the descending colon, the omentum, the distal pancreas, the spleen, and the distal duodenum (D4). Post operative course was uncomplicated.

Pathologic evaluation revealed a highly cellular neoplasm displaying areas of necrosis. The neoplastic cells were arranged in sheets and displayed nested perivascu- lar growth. They contained variable amounts of eosinophilic to vacuolated to amphophilic granular cytoplasms, and their nuclei were pleomorphic, containing prominent nucleoli and displaying mitotic activity. Large unidentifiable cells were also present. Multiple immunohistochemical staining revealed that Ki-67 marked 40\% - 50\% of nuclei. Immunostains were negative for pankeratin, synaptophysin, chromogranin, inhibin, calretinin, CD117, CD34, S-100, melan-A, HMB-45, EMA, desmin, smooth muscle actin, and CD99. This was felt to be consistent with a high grade undifferentiated sarcoma.

\section{Discussion}

Meningiomas are the second most common primary neoplasms of the central nervous system [1], but are overall rare with an annual incidence of approximately 2 cases per 100,000 individuals [2]. The most frequent genetic mutation involved in meningiomas is an inactivation mutation in the neurofibromatosis 2 gene on chromosome 22 [3]. Other genes associated with meningioma include the MN1 gene and the PTEN gene [3,4]. The majority of meningiomas are benign, however they can have malignant potential [4]. Meningiomas are well visualized with contrast CT or MRI with gadolinium since they are extra-axial and vascularized [1].

The classification of meningiomas is based on the World Health Organization classification system. Grade I is considered benign, Grade II are atypical, and Grade III are anaplastic [6]. Treatment with close observation and serial imaging studies can be done in small asymptomatic tumors, but is not recommended in symptomatic tumors [7]. These often require surgery with complete surgical excision being the standard treatment [8].

Retroperitoneal sarcomas are rare mesenchymal neoplasms accounting for only $1 \%$ - $2 \%$ of all solid malignnancies, and an overall incidence of 0.4 per 100,000 individuals [9]. The most common histological subtypes are liposarcomas and leiomyosarcomas [9]. Pleomorphic sarcomas are a rare high grade undifferentiated subtype. Patients with retroperitoneal sarcomas typically present late, since these tumors arise in the large potential spaces of the retroperitoneum, and are therefore asymptomatic until they are very large [9].

The staging of retroperitoneal sarcomas is based on the American Joint Committee Staging System which is based on the TMN classification [9]. Regardless of the histological subtype, the prognosis is defined by the grade of the sarcoma [10]. After histological grade, longterm survival is most dependant on the completeness of the resection, as complete resection provides the best chance for long-term survival [10]. In all cases, the surgical goal should be complete resection of the tumor with 
a microscopically negative margin (R0) [10]. Due to their large size at presentation, viscera are often involved by the tumor and visceral resection is therefore often required during tumor extirpation [10]. Nephrectomy followed by colectomy are the most commonly performed procedures during resection of retroperitoneal sarcomas [11].

The role of neoadjuvant, adjuvant, and radiation therapy remains unclear. The vast majority of patients will experience local recurrence [10]. With locally recurrent disease, complete surgical resection should remain the goal [10]. If complete resection is not believed to be feasible, systemic therapy should be considered with surgical exploration reserved for palliative intent [12].

It is highly unusual for a 39 year old otherwise healthy patient to present with two rare, seemingly unrelated tumors. It is our suspicion that there is a genetic component involved, however to date no association has been described in the literature between meningiomas and extracranial sarcomas.

\section{Conclusions}

Meningiomas are rare tumors, often associated with the Neurofibromatosis 2 gene. The majority are benign, however they can have malignant potent. Retroperitoneal sarcomas are rare mesenchymal tumors. They are most commonly liposarcomas or leiomyosarcomas. Pleomorphic sarcomas are a high grade undifferentiated subtype. The mainstay of treatment is complete surgical resection with microscopically negative margin. Resection of ad- jacent organs is often necessary to achieve negative mar- gins.

There has been no published genetic association between intracranial meningiomas and extracranial sarcomas. Besides being the first report of a retroperitoneal sarcoma occurring as a second metachronous neoplasm following an intracranial meningioma, we suspect that there is a genetic component involved. Further studies may indicate a genetic mutational link.

\section{REFERENCES}

[1] M. P. Buetow, P. C. Buetow and J. G. Smirniotopoulos, “Typical, Atypical, and Misleading Features in Meningioma,” Radiographics, Vol. 11, No. 6, 1991, pp. 10871106.

[2] M. Rohringer, G. R. Sutherland, D. F. Louw and A. A.
Sima, "Incidence and Clinicopathological Features of Meningioma,” Journal of Neurosurgery, Vol. 71, No. 5, 1989, pp. 665-672. doi:10.3171/jns.1989.71.5.0665

[3] K. Striedinger, S. R. Vandenberg, G. S. Baia, et al., "The Neurofibromatosis 2 Tumor Suppressor Gene Product, Merlin, Regulates Human Mingioma Cell Growth by Signaling through YAP,” Neoplasia, Vol. 10, No. 11, 2008, pp. 1204-1212.

[4] F. J. Staal, R. B. Van Der Luijt, M. R. Baert, et al., “A Novel Germline Mutation of PTEN Associated with Brain Tumors of Multiple Lineages," British Journal of Cancer, Vol. 86, No. 10, 2002, pp. 1586-1591. doi:10.1038/sj.bjc.6600206

[5] B. J. Goldsmith, W. M. Wara, C. B. Wilson, et al., "Postoperative Irradiation for Subtottaly Resected Mingiomas. A Restrospective Analysis of 140 Patients Treated from 1967 to 1990,” Journal of Neurosurgery, Vol. 80, No. 2, 1994, pp. 195-201. doi:10.3171/jns.1994.80.2.0195

[6] G. Wrobel, P. Roerig, F. Kokocinski, et al., "Microarrawy-Based Gene Expression Profiling of Benign, Atypical and Anaplastic Meningiomas Identified Novel Genes Associated with Meningioma Progression,” International Journal of Cancer, Vol. 114, No. 2, 2005, pp. 249-256. doi:10.1002/ijc.20733

[7] W. C. Olivero, et al., "The Natural History and Growth Rate of Asymptomatic Meningiomas: A Review of 60 Patients," Journal of Neurosurgery, Vol. 83, No. 2, 1995, pp. 222-224. doi:10.3171/jns.1995.83.2.0222

[8] C. Maros, M. Hassler, K. Roessler, et al., "Meningioma," Critical Reviews in Oncology/Hematology, Vol. 67, No. 2, 2008, pp. 153-171. doi:10.1016/j.critrevonc.2008.01.010

[9] I. Francis, R. Cohan and D. Varma, "Sondak V. Retroperitoneal Sarcomas,” Cancer Imaging, Vol. 5, No. 1, 2005, pp. 89-94. doi:10.1102/1470-7330.2005.0019

[10] J. Mullinax, J. Zager and R. Gonzalez, “Current Diagnosis and Management of Retroperitoneal Sarcoma," Cancer Control, Vol. 18, No. 3, 2011, pp. 177-187.

[11] S. Bonvalot, M. Rivoir, M. Castaing, et al., "Primary Retroperitoneal Sarcomas: A Multivariate Analysis of Surgical Factors Associated with Local Control,” Journal of Clinical Oncology, Vol. 27, No. 1, 2009, pp. 31-37. doi:10.1200/JCO.2008.18.0802

[12] R. Daylami, A. Amiri, B. Goldsmith, et al., "Inferior Vena Cava Leiomyosarcomas: Is Reconstruction Necessary after Resection?” Journal of the American College of Surgeons, Vol. 210, No. 2, 2010, pp. 185-190. doi:10.1016/j.jamcollsurg.2009.10.010 